\title{
FoodFinder: developing a rapid low-cost crowdsourcing approach for obtaining data on meal size from meal photos
}

\author{
L. Johnson ${ }^{1,3}$, C.Y. England ${ }^{1,3}$, P. Laskowski ${ }^{2}$, P.R. Woznowski ${ }^{2}$, L. Birch ${ }^{3}$, \\ J.P. Hamilton-Shield ${ }^{3}$, D.A. Lawlor ${ }^{4}$, I. Craddock ${ }^{2}$ and A. Skinner ${ }^{4}$
}

${ }^{1}$ Centre for Exercise, Nutrition and Health Science, University of Bristol, Bristol, BS8 1TZ, ${ }^{2}$ Faculty of Engineering, University of Bristol, Bristol, BS8 $1 \mathrm{UB},{ }^{3}$ NIHR Bristol Biomedical Research Unit in Nutrition, University of Bristol, Bristol, BS2 8AE and ${ }^{4}$ Medical Research Council Integrative Epidemiology Unit, University of Bristol, Bristol, BS8 $2 B N$

Capturing meal images using mobile phone cameras is a promising alternative to traditional dietary assessment methods. Acquiring photos is reasonably simple but nutritional content analysis of images is a major challenge. Automated food identification and portion size assessment is computationally and participant intensive; relying on participant feedback for accuracy ${ }^{(1)}$. Dietitian analysis of photos is accurate but time-consuming and expensive ${ }^{(2)}$. Crowdsourcing could offer a rapid low-cost alternative by utilising the life-long experience that all humans have in food identification. Previous crowdsourcing methods include the Eatery app, which produces a simple 11-point 'healthiness' scale for each meal ${ }^{(3)}$ and the PlateMate system, which creates a list of all individual foods with portion sizes, energy and macronutrient content ${ }^{(4)}$. While the Eatery produces limited and subjective data on meal content, PlateMate represents a complex integrated system of multiple tasks requiring on average 25 workers, costing $£ 2.75$ and taking 90 min per image. For feasible data-capture in a large-scale longitudinal studies, crowdsourcing data from meal photos needs to be cheaper and quicker. We aimed to develop a simpler task and tested it's feasibility for crowdsourcing dietary data.

FoodFinder, a single task for identifying food groups and portion sizes, developed using Qualtrics (www.qualtrics.com/), and linked to the Prolific Academic (https://prolific.ac/) crowdsourcing platform for recruitment and reimbursement of a UK crowd. Thirty meal photos with measured total meal weight (grams) were analysed by a dietitian and crowds ranging in size from 5 to 50 people. The difference between actual meal weight (the gold-standard) and total meal weight estimated by different sized crowds and ratings by a dietician were compared to each other. To establish group consensus crowd estimates were weighted by majority agreement ${ }^{(5)}$. Bland-Altman analysis assessed agreement with actual meal weight.

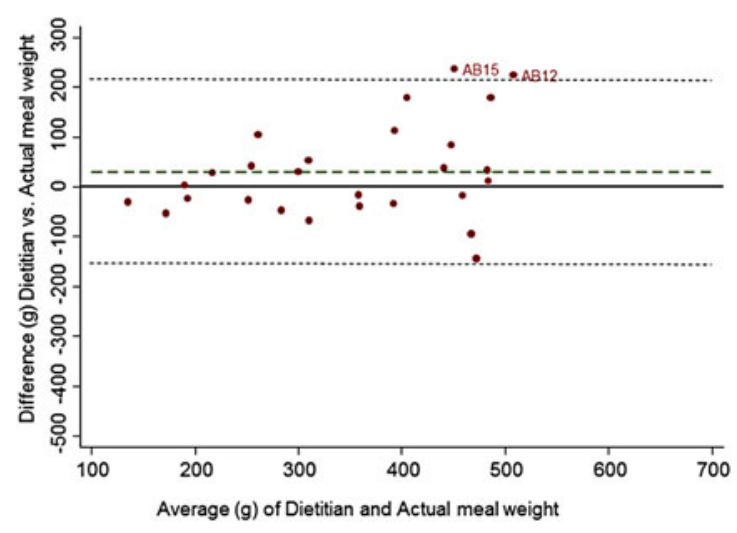

Fig. 1

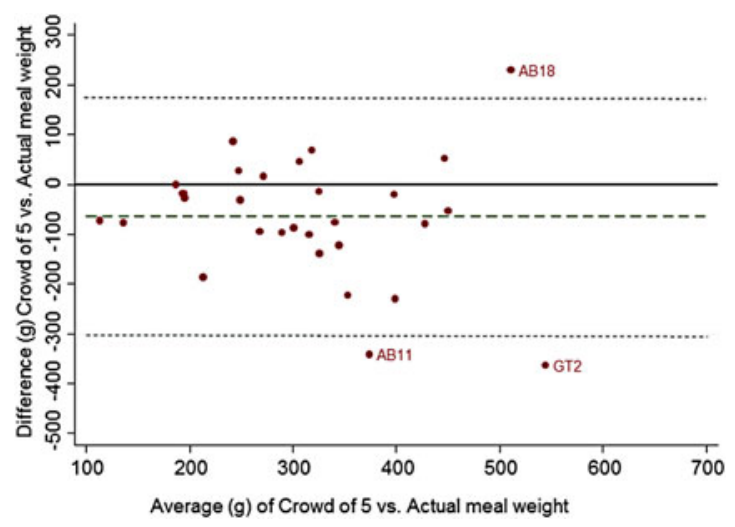

Fig. 2

A crowd of 5 people underestimated true meal weight by $63 \mathrm{~g}$, equating to $15 \%$ of actual meal weight with limits of agreement (LOA) from -299 to $174 \mathrm{~g}$. In comparison experts overestimated by $28 \mathrm{~g}$ equating to $9 \%$ of actual meal weight with LOA -158 , $214 \mathrm{~g}$. With a crowd of 5 people, crowdsourcing cost $£ 3.35$ and took a mean 2 mins 55 sec (SD 2 min 6 sec) per image. A crowd of 50 had similar accuracy and limits of agreement $(-65 \mathrm{~g} \mathrm{LOA}-278,149 \mathrm{~g})$ but was more expensive. Further development of FoodFinder is required to make rapid low-cost analysis of meal photos via crowdsourcing a feasible method for assessing diet.

This work was supported by a catalyst award from the Elizabeth Blackwell Institute for Health Research, which is co-funded by the University of Bristol and an Institutional Strategic Support Fund from the Wellcome Trust.

1. Boushey CJ, Kerr DA, Schap TE, et al. (2012) Eur J Clin Nutr 6, 648.

2. Martin CK, Correa JB, Han H, et al. (2012) Obesity (Silver Spiring) 20, 891-9.

3. Turner-McGrievy GM, Helander EE, Kaipainen K, et al. (2014) J Am Med Inform Assoc. e1. e112-9.

4. Noronha J, Hysen E, Zhang H, et al. (2011) Proc UIST 1-12.

5. Zhai Z, Hachen D, Kijewski-Correa T, et al. (2012) $45^{\text {th }}$ Int Conf Sys Sci, doi:10.1109/HICSS.2012.151 